ACCEPTED MANUSCRIPT

\title{
Motion control in free-standing shape-memory actuators
}

To cite this article before publication: Alberto Francisco Belmonte et al 2018 Smart Mater. Struct. in press https://doi.org/10.1088/1361$\underline{665 X / a a c 278}$

\section{Manuscript version: Accepted Manuscript}

Accepted Manuscript is "the version of the article accepted for publication including all changes made as a result of the peer review process, and which may also include the addition to the article by IOP Publishing of a header, an article ID, a cover sheet and/or an 'Accepted Manuscript' watermark, but excluding any other editing, typesetting or other changes made by IOP Publishing and/or its licensors"

This Accepted Manuscript is ( 2018 IOP Publishing Ltd.

During the embargo period (the 12 month period from the publication of the Version of Record of this article), the Accepted Manuscript is fully protected by copyright and cannot be reused or reposted elsewhere.

As the Version of Record of this article is going to be / has been published on a subscription basis, this Accepted Manuscript is available for reuse under a CC BY-NC-ND 3.0 licence after the 12 month embargo period.

After the embargo period, everyone is permitted to use copy and redistribute this article for non-commercial purposes only, provided that they adhere to all the terms of the licence https://creativecommons.org/licences/by-nc-nd/3.0

Although reasonable endeavours have been taken to obtain all necessary permissions from third parties to include their copyrighted content within this article, their full citation and copyright line may not be present in this Accepted Manuscript version. Before using any content from this article, please refer to the Version of Record on IOPscience once published for full citation and copyright details, as permissions will likely be required. All third party content is fully copyright protected, unless specifically stated otherwise in the figure caption in the Version of Record.

View the article online for updates and enhancements. 


\title{
Motion control in free-standing shape-memory actuators
}

\author{
Alberto Belmonte ${ }^{1}$, Giuseppe C. Lama ${ }^{2,3}$, Pierfrancesco Cerruti ${ }^{3}$, Veronica Ambrogi ${ }^{2,3}$, \\ Xavier Fernández-Francos ${ }^{4}$, Silvia De la Flor ${ }^{1 *}$
}

${ }^{1}$ Department of Mechanical Engineering, Universitat Rovira i Virgili, Av. Països Catalans, 26, 43007, Tarragona (Spain), albertofrancisco.belmonte@urv.cat

${ }^{2}$ Department of Chemical, Materials and Production Engineering, University of Naples

“Federico II", Piazzale Tecchio, 80, 80125, Napoli (Italy), giuseppecesare.lama@live.it and ambrogi@unina.it

${ }^{3}$ Institute for Polymers, Composites and Biomaterials, National Council of Research of Italy, Via Campi Flegrei, 34, 80078, Pozzuoli (NA) (Italy), cerruti@ipcb.cnr.it

${ }^{4}$ Thermodynamics Laboratory ETSEIB, Universitat Politècnica de Catalunya, Av. Diagonal, 647, 08028, Barcelona (Spain), xavier.fernandez@mmt.upc.edu

* Correspondence: silvia.delaflor@urv.cat; Tel.: +34-977558839; Fax: +34-977559602

\begin{abstract}
In this work, free-standing shape-memory thermally triggered actuators are developed by laminating "thiol-epoxy"-based glassy thermoset (GT) and stretched liquid-crystalline network (LCN) films. A sequential curing process was used to obtain GTs with tailored thermomechanical properties and network relaxation dynamics, and also to assemble the final actuator. The actuation extent, rate and time were studied by varying the GT and the heating rate in thermoactuation with an experimental approach. The results demonstrate that it is possible to tailor the actuation rate and time by designing GT materials with a glass transition temperature close to that of the liquid-crystalline-to-isotropic phase transition of the LCN, thus making it possible to couple the two processes. Such coupling is also possible in rapid heating processes even when the glass transition temperature of the GT is clearly lower than the isotropization temperature of the LCN, depending on the network relaxation dynamics of the GT and the presence of thermal gradients within the actuators. Interestingly, varying the GT network relaxation dynamics does not affect the actuation extent. As predicted by the analytical model developed in our previous work, the modulus of the GT layer is mainly responsible for the actuation extent. Finally, to demonstrate the enhanced control of the actuation, specifically designed actuators were assembled in a three-dimensional actuating device able to make complex motions (including "S-type" bending). This approach makes it possible to engineer advanced functional materials for application in self-adaptable structures and soft robotics.
\end{abstract}

KEYWORDS: Free-standing; shape-shifting; actuator; liquid-crystalline network; dual-curing; experimental study. 


\section{INTRODUCTION}

Actively moving polymers (AMPs) are a class of stimuli-responsive materials that can undergo large physical changes upon stimulation. According to the definition given by Lendlein and Behl in [1], AMPs can be categorized into shape-memory polymers (SMPs) and shape-changing polymers (SCPS) depending on the transformation mechanisms: SMPs are triggered by the shape-memory effect (SME) [2-4], whereas the SCP response is related to the shape-changing effect (SCE) $[5,6]$. The main difference is that SMPs require a reiterated programming process and SCPs require constant stimulation to achieve transformation processes. The SME relies on the softening/freezing transition in glassy or semi crystalline networks, which is generally a glass or melting transition. The softening of the network allows a deformation process towards a temporary shape that is eventually fixed through the vitrification or crystallization process thanks to the reduced network mobility.

Many parameters, such as the crosslinking density, the crystallisable chains and the chain flexibility, have a crucial effect on the dynamics of the network transitions, and hence on the SME $[7,8]$. Among the different SMPs, epoxy-based glassy networks stand out due to their excellent shape-recovery and -fixation performances arising from their well-defined networks and the drastic structural changes that take place during the glass transition. Different curing agents have been employed to overcome the inherent fragility and low deformability of the epoxy networks while preserving their ultimate strength, i.e. increasing the rubbery modulus by using anhydrides [9], enhancing deformability by using amines [10] and improving both the deformability and the resistance at break by using flexible thiols [11,12]. Another approach is incorporating hyperbranched polymers as polymeric modifiers of SMP because of their attractive properties resulting from the combination of a globular and densely branched structure and the possibility of tailoring their physical-chemical compatibility with a variety of resins by modification of their end functional groups. The resulting heterogeneous network obtained substantially enhanced their mechanical properties, especially stress and strain at break. This effect enabled one of the most promising applications of these materials, namely thermomechanical actuators, which work under constant stress or strain [13]. Other approaches, such as tailoring the viscoelastic response at the deformation temperature and imposing isothermal recovery conditions, have been employed to enhance the mechanical limits and control the dynamics of the recovery process $[14,15]$.

Liquid-crystalline networks (LCNs) consisting in lightly crosslinked polymers containing ordered phases called liquid-crystalline domains are a good example of SCPs as they can react mechanically to the appropriate stimulus through the reversible anisotropic-to-isotropic transition, that is, the formation and disorder of the liquid-crystalline domains. Generally, thermomechanical stimulation causes the shape-changing effect in SCPs by means of a stressalignment of the LC domains into a highly oriented monodomain structure during the isotropicto-anisotropic transition. The liquid-crystallinity, crosslinking density, the structure of the mesogenic compounds and their alignment properties have been reported to be the crucial parameters for controlling the shape-change extension and the stress threshold to trigger the shape-change. Kessler et al. [16] reported an easy approach for controlling these parameters by using carboxylic acids of different aliphatic chain lengths. Burke et al. [17] found an increase in 
the shape-change extension and a decrease in the stress threshold when the crosslinking density in main-chain LC elastomers was increased. Interestingly, Lama et al. [18] improved the stress threshold by incorporating carbon nanotubes as guest agents. Unfortunately, the fact that SCPs require the constant application of stress considerably narrows their application range.

In order to overcome this drawback, researchers have focused their efforts on optimizing the bidirectional actuation in LCNs through the interaction of combined network structures to achieve the required mechanical stimulus for free-standing bidirectional actuation $[19,20]$. Two lines of research have arisen: the first line involves the internal incorporation of an elastic network in the SCP. Anthamatten et al. [21] demonstrated this internal incorporation by using dual-cured networks via stress-induced alignment after the $1^{\text {st }}$ curing process. A unidirectional actuation of $15 \%$ was achieved. Similarly, Yakacki et al. [22] enhanced the actuation extent up to $110 \%$ by using two-stage (thermal/UV) thiol-acrylate systems. The second line is based on the external attachment of an elastic material to the SCP. Westbrook et al. [23] embedded a stretched poly(cyclooctene)-based LCN into an elastomeric matrix through thermal curing of the latter, which led to small reversible flexural actuation by heating-cooling procedures. The resulting material could bend and unbend upon heating or cooling with a flexural actuation of 6 $\mathrm{mm}$.

As stated above, not only is bidirectional motion necessary, but also complex shape designs. Emerging techniques like 3D printing have been used as a solution for achieving complex spatial designs as demonstrated in [24]; however, these techniques are still limited to certain materials. Our research group demonstrated that sequential dual-curing "thiol-epoxy" systems can be used to achieve thermally-triggered free-standing bidirectional shape-memory actuators with enhanced control of the motion design by carefully designing the device [25]. Multi-layer assemblies were prepared by external bonding of LCN and epoxy-based thermoset (glassy thermoset, GT) layers of controlled thickness. The use of partially-cured GT layers in the assembly process and their subsequent post-curing, following a sequential dual-curing scheme, enhanced the inter-layer adhesion [26-28]. Solid but highly deformable intermediate, partially cured materials can be obtained by appropriately choosing the appropriate "thiol-epoxy" ratio and the thiol and epoxy compounds. This also affects the final $T_{g}$ of the GT material. In general terms, the final $T_{g}$ of the GT should be lower than the $T_{\text {iso }}$ of the LCN. Therefore, the assembled actuator will actuate upon the elastic response of the GT that generates the necessary force to promote the alignment of the LC domains during the isotropic-to-anisotropic transition and permits the elastic shrinkage of the LCN during the anisotropic-to-isotropic transition. With this methodology not only is it possible to efficiently tailor the properties of the GT material, hence the actuation of the device, but also to design complex 3D devices thanks to the processing of a highly deformable solid state. The possibility to cure solid and stable materials for the assembling process reduces the limitations of moulding techniques required for liquid-like mixtures. Moreover, it makes it possible to process complex shapes after the $2^{\text {nd }}$ curing stage, such as, curved designs, in an easy and controlled manner as demonstrated in our previous work [27].

In this work, the effect of the thermomechanical properties and network relaxation of the GT material on the actuation extent, time and rate has been analysed. GT materials with different moduli, crosslinking densities, $\mathrm{T}_{\mathrm{g}}$ and network relaxation dynamics were obtained by changing the thiol and epoxy compounds and their ratio. The cyclic response was analysed in detail and the effect of the GT properties and relaxation dynamics were studied under non-isothermal 
conditions at different heating rates. In addition, we demonstrate the possibility of engineering a 3D actuating device by assembling specifically designed actuators with different actuation extents, times and rates. The device is assembled in one single piece, taking advantage of the enhanced processing of the GT material in the intermediate stage. The complex actuation of the resulting 3D device was recorded, analysed through visual frames, and compared with the predictions of the theoretical model developed in our previous work [25].

\section{EXPERIMENTAL PROCEDURES}

\subsection{Materials and Methods}

\subsubsection{Epoxy-based thermoset (GT)}

Diglycidyl ether of bisphenol A (DGEBA, GY240, Hutsman) with an epoxy equivalent weight of $182 \mathrm{~g} / \mathrm{eq}$. and $\mathrm{EPON}^{\mathrm{TM}}$ resin SU8 with an epoxy equivalent average weight of $215 \mathrm{~g} / \mathrm{eq}$. were used as epoxy resins. Pentaerythritol tetrakis(3-mercaptopropionate) (\$4) with a thiol equivalent weight of $122.17 \mathrm{~g} / \mathrm{eq}$. and trimethylolpropane tris (3-mercaptopropionate) (S3) with a thiol equivalent weight of $132.85 \mathrm{~g} / \mathrm{eq}$. were purchased from Sigma-Aldrich and used as curing agents in an under-stoichiometric proportion with respect to the epoxy groups in the system. 1methylimidazole (1Ml, sigma-Aldrich, $82.1 \mathrm{~g} / \mathrm{mol}$ ) was used as catalyst in a proportion of $1 \mathrm{phr}$ (parts of catalyst per hundred parts of the whole mixture). The molecular structures of all the compounds are presented in the Supporting/Information. DGEBA was dried overnight under vacuum at $80{ }^{\circ} \mathrm{C}$ before use. The other reagents were used as received without further purification. Three different "thiol-epoxy" systems were studied according to our previous works $[26,27]$ : DGEBA-S3-1MI, DGEBA-S4-1MI and (DGEBA-SU8)-S3-1MI with a DGEBA-SU8 molar proportion of $(7: 3)$. As it was demonstrated, these systems lead to sequential dual-curing processing when under-stoichiometric thiol equivalents are used with respect to the epoxy groups: the "thiol-epoxy" polycondensation ( $1^{\text {st }}$ curing stage) takes place at a low temperature $\left(50^{\circ} \mathrm{C}\right)$ during 1 hour and 45 minutes in the presence of the catalyst, leading to the intermediate, stable material, whereas the homopolymerization of the remaining epoxy groups ( $2^{\text {nd }}$ curing stage) is triggered at a higher temperature $\left(T>90^{\circ} \mathrm{C}\right)$ in the presence of the catalyst that is regenerated during the $1^{\text {st }}$ curing stage.

\subsubsection{Liquid-crystalline network (LCN)}

An epoxy-based mesogenic compound was synthesized by epoxidation of a commercial diol (4,4'-dihydroxybiphenyl, DIF, 97\% purity, $186.21 \mathrm{~g} / \mathrm{mol}$ ), using epichlorohydrin (99\% purity, $1.183 \mathrm{~g} / \mathrm{ml}, 92.52 \mathrm{~g} / \mathrm{mol}$. Sigma-Aldrich), isopropanol and sodium hydroxide. A commercial dicarboxylic acid (1,8-octanedicarboxylic acid, sebacic acid, 98\% purity, $202.25 \mathrm{~g} / \mathrm{mol}$, SigmaAldrich) was used as curing agent. Trycaprylyl methylammonium chloride (Sigma-Aldrich) was used as catalyst in the reaction.

The LCN was prepared as explained in our previous work [29]: first the mesogenic compound and the acid were melted at $180^{\circ} \mathrm{C}$ and homogenized with magnetic stirring, then the catalyst was added and the mixture was poured into a Teflon-covered glass mould with $250 \mu \mathrm{m}$ spacing. Films of $8 \times 8 \mathrm{~cm}^{2}$ with thickness of $250 \mu \mathrm{m}$ were obtained. 


\subsubsection{Preparation of the actuators}

The preparation of the LCN-GT actuator (illustrated in Figure 1) was carried out as explained in our previous work [25]: the GT intermediate material was prepared in the form of films with different thicknesses by pouring the initial "thiol-epoxy" mixture containing all the reactants, including the catalyst, in between Teflon-coated glass plates and carrying out the $1^{\text {st }}$ curing process (see Figure 1(a)) as explained above. The LCN layer was programmed from a piece of the previously prepared LCN film (see Figure 1(b)) as follows: First it was heated from room temperature $\left(\mathrm{T}_{\text {room }}=25^{\circ} \mathrm{C}\right)$ to $\mathrm{T}_{\text {iso }}+20^{\circ} \mathrm{C}$ to elastically deform the network at a constant stress level $\left(\sigma_{p}\right)$, corresponding to a predetermined $\%$ of the stress at break $\left(\sigma_{b}=780 \mathrm{kPa}\right)$. Then, it was cooled down to induce the elongation effect caused by the formation and alignment of the LC domains in the stress direction and promoted by the soft elasticity phenomenon of the LCN. The stress applied was released and the corresponding programmed strain $\left(\varepsilon_{\mathrm{p}}\right)$ was stabilized at a low temperature (see the strain-temperature graphic and the LCN programmed layer in Figure 1(b)). Once both the LCN and GT intermediate materials had been prepared, the actuator was assembled layer-by-layer in a sandwich-like configuration (see Figure 1(c)). To do this, the LCN programmed layer and GT materials were first cut to fit specific dimensions for a beam-like design of $20 \times 3.5 \mathrm{~mm}^{2}$ (length $\mathrm{x}$ width). The total thickness of the assembled actuator was defined through the volume fraction $\left(\mathrm{V}_{\mathrm{F}}\right)$, a parameter that considers the total thickness of the actuator in relation to the GT total thickness according to equation (1)Error! Reference source not found.. A thinner GT layer (layer $1, \mathrm{t}_{\mathrm{L} 1}=0.24 \mathrm{~mm}$ ) was placed on one side, the LCN layer (layer 2, $\mathrm{t}_{\mathrm{L} 2}<0.25$ after programming) was placed in the middle, and a thicker GT layer (layer 3 ) was placed on the other side with the corresponding thickness to fit the specific volume fraction. This configuration makes it possible to obtain a controlled bending actuation towards layer 1 due to the lower thickness $\left(t_{L_{1}}<t_{L_{3}}\right.$ ) and enhances the adhesion with the LCN layer from both surfaces. An enlarged schematic representation of the final actuator deflection is depicted in Figure 1(d). According to the theory of elasticity for multilayered beams subject to built-in stresses [30,31], with this configuration, the final actuator will bend downwards with respect to the thinner GT layer (layer 1) due to the contraction of the LCN, as illustrated in Figure 1(d).

$$
v_{F}=\frac{V_{G T}}{V_{t o t}}=\frac{t_{G T}}{t_{t o t}}=\frac{t_{L 1}+t_{L 3}}{t_{L 1}+t_{L 2}+t_{L 3}}
$$




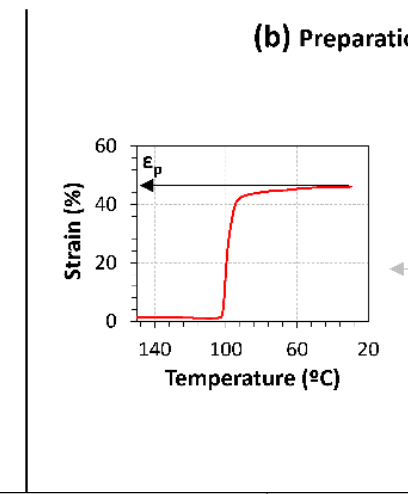

(c) Configuration of the actuator device after assembling
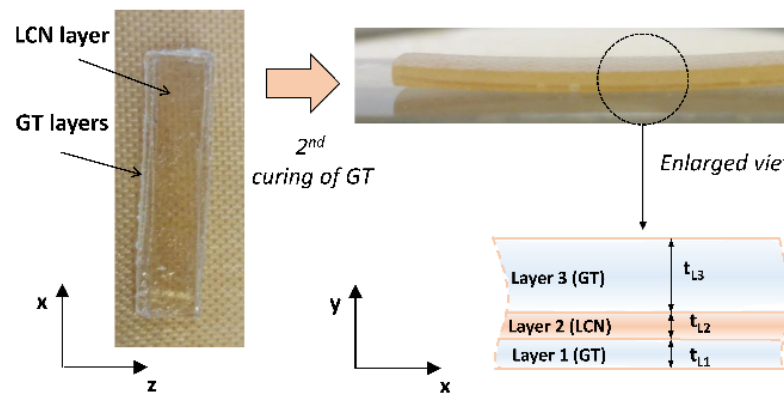

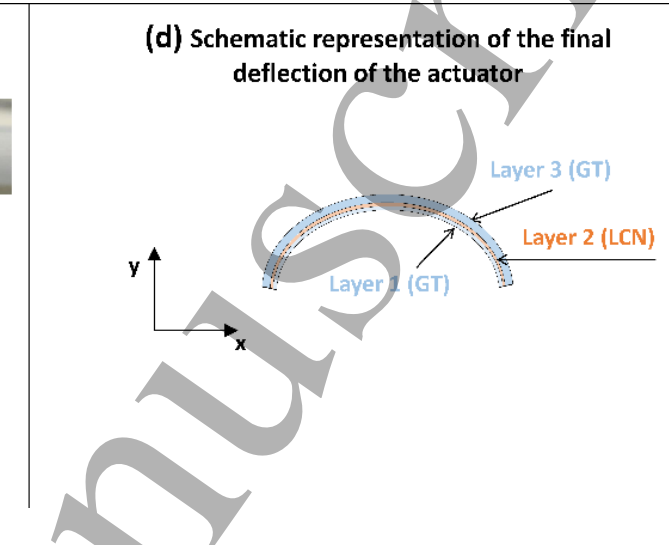

Figure 1. Preparation of the actuator device: (a) curing and processing of GT layers 1 and 3; (b) programming process of LCN layer 2. The strain-temperature curve used (extracted from our previous work [25]) to determine the level of programing is included; (c) assembled configuration of the device with an enlarged schematic representation of the layers; and (d) illustration of the final actuation deflection.

Once the actuator configuration is built, the $2^{\text {nd }}$ curing stage of the GT material is carried out by placing the actuator in between Teflon glass plates, applying a small constraining force to prevent the LCN shrinkage, and following a specific temperature programme. In our previous work [25], a temperature of $90^{\circ} \mathrm{C}$ was used to limit the LCN premature shrinkage; however, GT materials with glass transition temperatures close to or equal to $\mathrm{T}_{\text {iso }}\left(120^{\circ} \mathrm{C}\right)$ were used in this work. Therefore, to allow completion of epoxy homopolymerization ( $2^{\text {nd }}$ curing stage), the following curing procedure was used: 3 hours at $90{ }^{\circ} \mathrm{C}$ plus 1 hour at $110{ }^{\circ} \mathrm{C}$ plus 1 hour at 135 ${ }^{\circ} \mathrm{C}$. The progressive increase of the curing temperature promotes the formation of the GT network in different steps, preventing the LCN from shrinking inside the actuator. At $90{ }^{\circ} \mathrm{C}$, the LCN remains stable, whereas part of the epoxy homopolymer is formed and bound to the LCN surface. LCN shrinkage to some extent cannot be avoided at the higher temperatures, but the LCN displacement is prevented through the adhesion with the homopolymer network already formed during the previous three hours at $90{ }^{\circ} \mathrm{C}$, and which reaches its ultimate structure after the higher temperature steps. The application of a constraining force through the glass plates avoids undesired bending of the whole actuator.

\subsection{Thermomechanical characterization of the GT materials}

Dynamic mechanical analyses were carried out using a Q800 DMA (TA Instruments) apparatus equipped with a 3-point-bending clamp. The experiments were performed at an oscillation frequêncy of $1 \mathrm{~Hz}$ with a strain amplitude of $0.1 \%$ from $35^{\circ} \mathrm{C}$ up to $180^{\circ} \mathrm{C}$ at a heating rate of 3 
${ }^{\circ} \mathrm{C} / \mathrm{min}$ in samples of the GT final material (after the $2^{\text {nd }}$ curing stage). "Thiol-epoxy" ratios ranging from 0.25 up to the stoichiometric proportion $(r=0.25,0.5,0.75$ and 1$)$ were analysed for the different systems studied (see Table 1). The $T_{\mathrm{g}}$ was determined from the peak of the tan $\delta$ curve, the modulus in the glassy and rubbery regions were obtained from the glassy and rubbery plateaus at $30{ }^{\circ} \mathrm{C}$ and $\mathrm{T}_{\mathrm{g}}+50{ }^{\circ} \mathrm{C}$. In addition, the tan $\delta$ peak value and the width at half-height (FWHM) were also determined as indicators of the network relaxation dynamics.

Table 1. Composition of the different systems under study

\begin{tabular}{|c|c|c|c|c|c|c|}
\hline System & "thiol-epoxy" ratio & $\begin{array}{l}\text { DGEBA } \\
\text { (\%wt.) }\end{array}$ & $\begin{array}{c}\text { SU8 } \\
\text { (\%wt.) }\end{array}$ & $\begin{array}{c}\text { S3 } \\
\text { (\%wt.) }\end{array}$ & $\begin{array}{c}\text { S4 } \\
\text { (\%wt.) }\end{array}$ & $\begin{array}{l}\text { 1-MI } \\
\text { (\%wt.) }\end{array}$ \\
\hline \multirow{4}{*}{ DGEBA-S3-1MI } & 0.25 & 83.7 & --- & 15.3 & 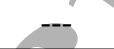 & 1.0 \\
\hline & 0.5 & 72.5 & --- & 26.5 & -- & 1.0 \\
\hline & 0.75 & 64.0 & --- & 35.0 & - & 1.0 \\
\hline & 1.0 & 57.2 & --- & 41.8 & -- & 1.0 \\
\hline \multirow{4}{*}{ DGEBA-S4-1MI } & 0.25 & 84.8 & --- & & 14.2 & 1.0 \\
\hline & 0.5 & 74.1 & --- & & 24.9 & 1.0 \\
\hline & 0.75 & 65.9 & --- & --- & 33.2 & 1.0 \\
\hline & 1.0 & 59.2 & -- & 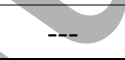 & 39.8 & 1.0 \\
\hline \multirow{4}{*}{ (DGEBA:SU8)-S3-1MI } & 0.25 & 56.0 & 28.4 & 14.6 & --- & 1.0 \\
\hline & 0.5 & 48.8 & 24.7 & 25.5 & --- & 1.0 \\
\hline & 0.75 & 43.3 & 21.9 & 33.8 & --- & 1.0 \\
\hline & 1.0 & 38.8 & 19.7 & 40.5 & --- & 1.0 \\
\hline
\end{tabular}

\subsection{Characterization of the actuation response}

The actuation response of the different samples was first stabilized through 20 consecutive "heating-cooling" cycles at constant heating and cooling rates of $2{ }^{\circ} \mathrm{C} / \mathrm{min}$. Afterwards, nine more cycles were carried out varying the heating rate from $0.2^{\circ} \mathrm{C} / \mathrm{min}$ up to $20^{\circ} \mathrm{C} / \mathrm{min}(0.2,0.5$, $1,2,3,5,10,15$ and $\left.20^{\circ} \mathrm{C} / \mathrm{min}\right)$, and keeping a cooling rate of $2{ }^{\circ} \mathrm{C} / \mathrm{min}$, to study the effect of the heating rate on the actuation. The thermal cycles were performed using the DMA equipment in force-controlled static mode with a custom-made clamp geometry (see Figure 2(a)). The sample was placed onto the flat surface, which produced an upwards displacement of the clamp upon the sample bending. The displacement of the moveable clamp was registered by applying a small force of $0.01 \mathrm{~N}$ centred in the actuator (the process is illustrated in Figure 2(a)). The actuation extent was determined considering the variation of the deflection, from zero (planar state) up to an arc of $180^{\circ}$, which is considered the maximum operationally effective angle. The instantaneous deflection can be measured directly as the displacement of the clamp $d(t)$ (depicted in Figure 2(a)), and the actuation extent as the quotient between the instantaneous deflection and the deflection corresponding to a bending angle of $180^{\circ}$. In equation (2), $d_{\max }$ is computed considering an arch of length $L\left(L\right.$, length of the actuator) of a semicircle of $180^{\circ}$ and radius $\mathrm{d}_{\max }$ :

$$
\text { Actuation }=\frac{d(t)}{d_{\max }}=\frac{d(t)}{L / \pi}
$$


(a)

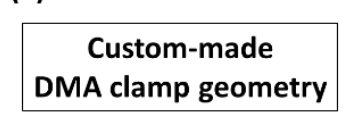

(b)

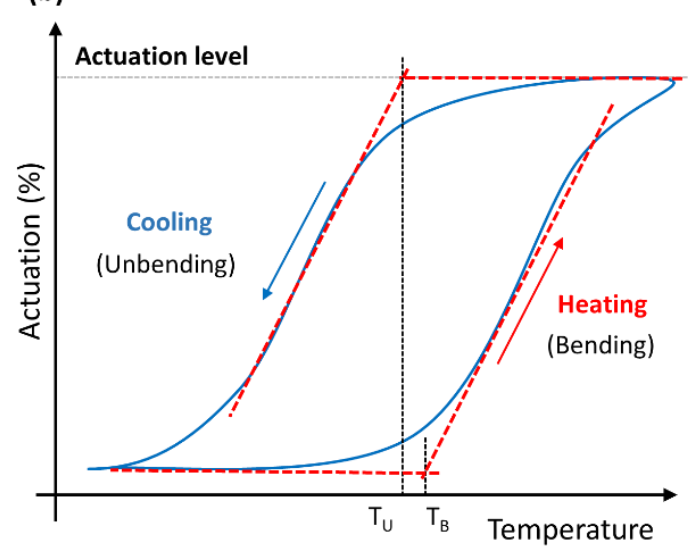

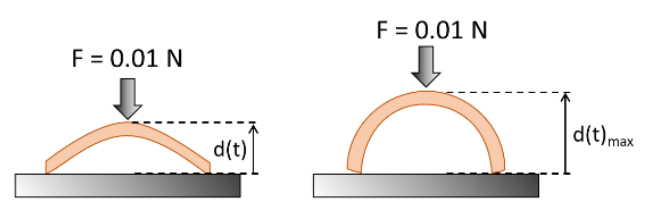

(c)

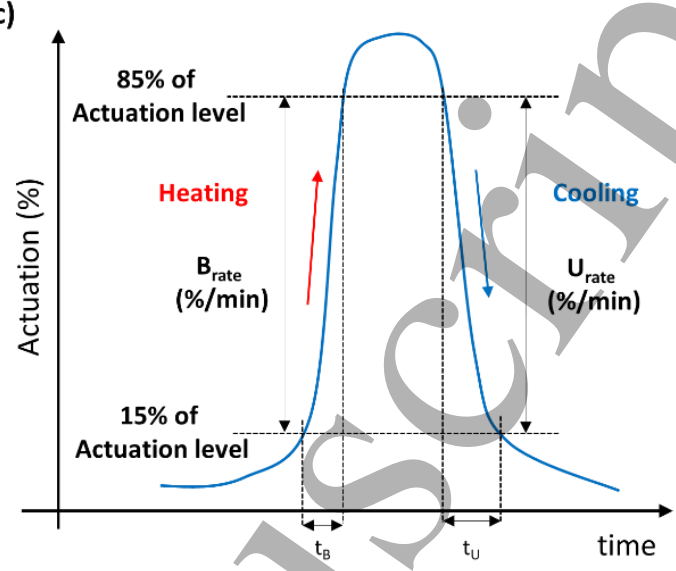

Figure 2. (a) Scheme of the DMA experiment set up to characterize the actuators; (b) scheme of the actuation-Temperature cycle, and (c) scheme of the actuation-time cycle.

Figure 2 shows the experimental characterization of the actuation with the DMA according to the explained experimental procedure. The Actuation-Temperature and Actuation-Time plots are represented in Figure 2(b) and 2(c). First, the actuation extent was defined as the maximum actuation achieved. From the hysteresis cycle in Figure 2(b), the temperatures corresponding to the beginning of the bending, $T_{B}$, and unbending, $T_{U}$, were determined as the onset point, that is, the intersection between the tangent lines to the hysteresis curve in the high slope and horizontal regions of the curves during the heating (bending) and cooling (unbending) processes, respectively, as indicated in Figure $2(b)$. The rate during the bending $\left(B_{\text {rate }}\right)$ and unbending $\left(\mathrm{U}_{\text {rate }}\right)$ processes were determined (in \%/min) from Figure 2(c) as the time needed to bend or unbend from $15 \%$ to $85 \%$ of the actuation strain. In addition, the time needed to achieve the same actuation range was considered separately $\left(t_{B}\right.$ and $\left.t_{U}\right)$, as indicated in Figure $2(c)$ ).

\subsection{Preparation of the $3 D$ actuating device}

A planar-to-3D transformation device was assembled to experimentally demonstrate the possibility of obtaining complex motion designs. As shown in Figure 3(a), the device consists of a central part made of GT to which six legs made of different actuators are anchored. All the actuators were made using the same stress programming level $\left(\sigma_{p}\right)$ for the LCN layer and different GTs to produce different motions. The actuation shape and direction of each leg was defined by the vertical positioning of the LCN layer in the sandwich structure, the structural properties of the GT material, and the combination of different actuators along the leg. To properly follow the configuration of the legs, the terminology "below" and "above" is used concerning the vertical positioning of the LCN layer in the sandwich structure (see also Fig. 8(c)). The shorter legs (Figure 3(a) legs 1-4) were made of one single LCN positioned below; thus, the actuation direction is the same for the four legs. However, each pair of legs (1-2 and 3-4 legs) was made of different GT material, hence different actuation extents are expected. In contrast, the longer legs were divided into two sections along the length (sections 5.1 / 5.2 and 6.1 / 6.2), 
(a) Mould device $(\mathrm{t}=\mathbf{1 . 2} \mathrm{mm})$

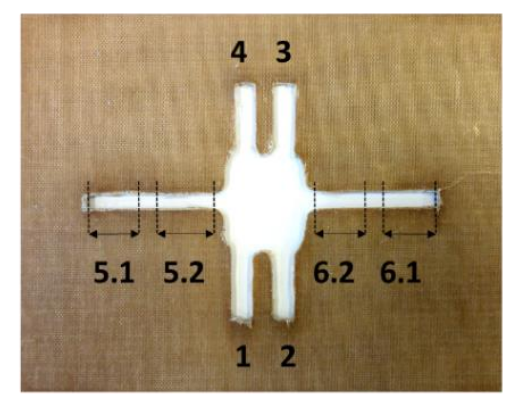

(b) Moulds assembling layers $\left(t_{L 1}=0.24 \mathrm{~mm}\right.$ and $\left.t_{L_{3}}=0.73 \mathrm{~mm}\right)$

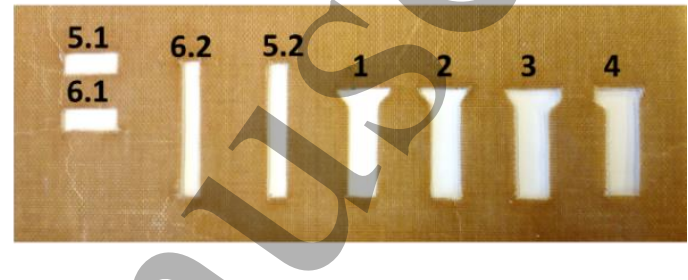

(c) Programmed LCN layers

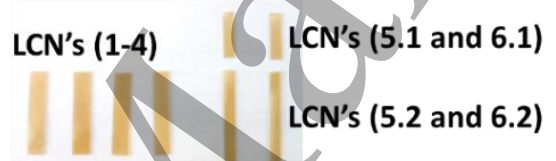

Figure 3. (a) Mould used for assembling the 3D device; (b) mould for the preparation of the GT-layers in the intermediate stage, and (c) LCN programmed layers.

The device was built in one single piece using the mould shown in Figure 3(a). Layers with different shapes of the GT material were prepared using the Teflon mould shown in Figure 3(b) to fit the design of each leg. Two different moulds were used: for layer 1 , a thinner mould $(t=$ $t_{L 1}$ ) and for layer 3 a thicker mould $\left(t=t_{\llcorner 3}\right)$. The $L C N$ layers were programmed as explained in the Materials and Methods Section and cut to fit the dimensions of each leg of the device (see Figure $3(\mathrm{c})$ ). Once the intermediate GTs and programmed LCN materials had been prepared, the device was mounted with a total thickness of $1.2 \mathrm{~mm}$ and a constant $\mathrm{v}_{\mathrm{F}}=0.8$ in all the actuators. All the LCN were programmed at the same extent and were positioned below or above depending on the leg as explained before. Accordingly, the GT thickness of the layer $\left(t_{L 3}\right)$ was defined to meet these requirements. Afterwards, a constraining force was applied using Teflon glass plates, and the same curing process as that adopted for a single actuator was followed: 3 hours at $90{ }^{\circ} \mathrm{C}$ plus 1 hour at $110^{\circ} \mathrm{C}$ plus 1 hour at $135^{\circ} \mathrm{C}$. Finally, the device was carefully polished to eliminate defects on the edges.

The individual response of all the actuators of the 3D device were previously characterized as explained in the previous section. The motion of the assembled device was tested in a thermal chamber under rapid heating and natural cooling processes from $50{ }^{\circ} \mathrm{C}$ up to $160{ }^{\circ} \mathrm{C}$. 20 cycles were performed to achieve stability and, once stabilized, subsequent cycles were recorded with a high-resolution camera. Frames of the device in motion at different times and positions are shown in Section 3.5. In addition, the bending angles on each actuator were determined using a 
computational tool and compared with the predicted angles using the analytical model developed in the previous work [25].

\section{RESULTS AND DISCUSSION}

\subsection{Thermomechanical properties of the GT systems}

The thermomechanical properties of the different GT systems studied were analysed by means of DMA analysis. The modulus, $E_{r}$, glass transition temperature, $T_{g}$ and tan $\delta$ breadth (FWHM and $\tan \delta_{\text {peak }}$ ) are shown in Figure 4 as a function of the "thiol-epoxy" ratio. As can be seen, the use of a thiol compound with higher functionality (S4 > S3) leads to the formation of network structures with higher rubbery modulus and $\mathrm{T}_{\mathrm{g}}$ (see the results for $r=1.0$ in Figure 4(a)). The higher functionality of $\mathrm{S} 4$ causes an increase in the crosslinking density, and thus an increase in $E_{r}$ according to the theory of elasticity [32]. In addition, the absence of the pendant ethyl chain in the S4 compound (the chemical structures are shown in the Supporting Information) promotes the formation of a more homogeneous and packed network, hindering the mobility of the network chains and therefore increasing the $T_{g}$. The incorporation of a highly functionalized epoxy resin (SU8) does not affect the rubbery modulus in the stoichiometric formulation, but increases the $\mathrm{T}_{\mathrm{g}}$ and considerably broadens the relaxation process as deduced by the increase in FWHM and decrease in the $\tan \delta_{\text {peak }}$ (see Figure 4(b)). The epoxy homopolymerization is extended by moving away from the stoichiometric mixture, that is, decreasing the "thiol-epoxy" ratio. Consequently, the crosslinking density and $\mathrm{E}_{\mathrm{r}}$ increase, and the relaxation process broadens due to the higher functionality of the epoxy homopolymerization in comparison with the thiol-epoxy polycondensation $\left(f_{4}>f_{2}\right)$.

(a)

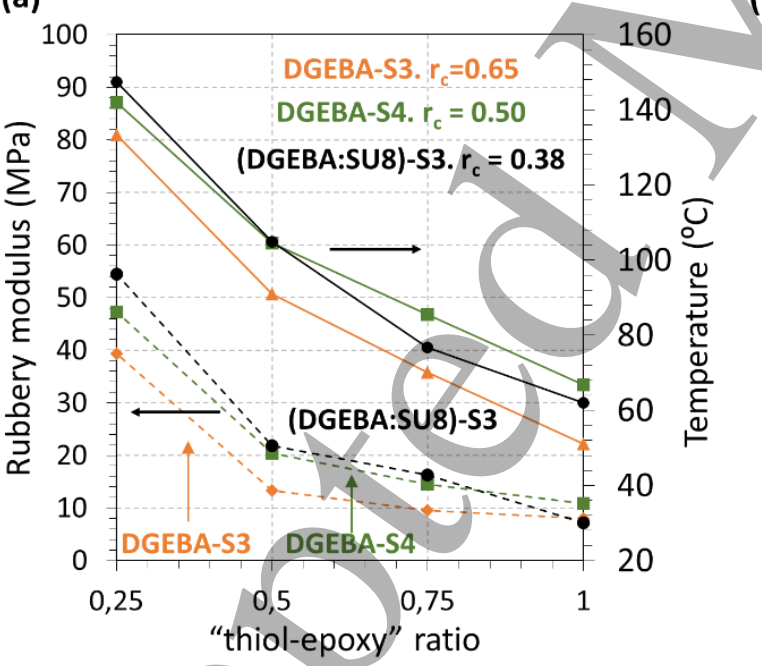

(b)

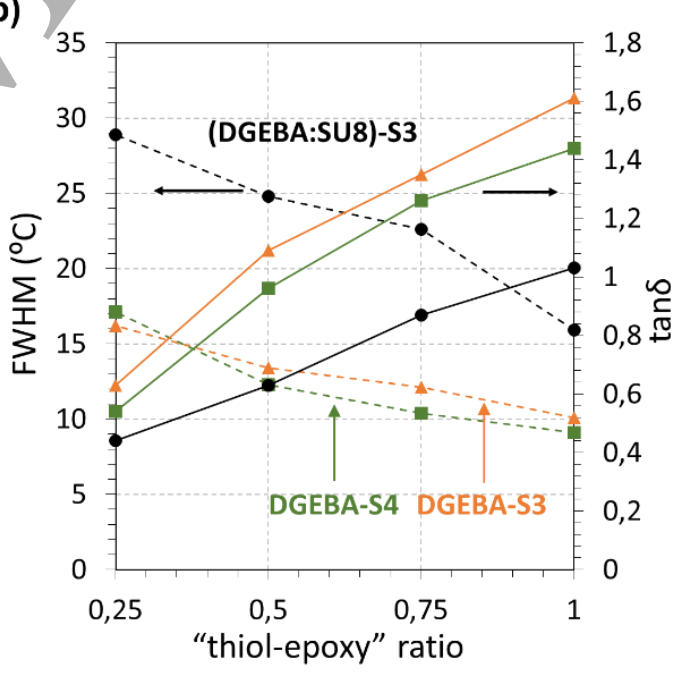

Figure 4. (a) FWHM and tan $\delta_{\text {peak, }}$ and (b) rubbery modulus and glass transition temperature as a function of the "thiol-epoxy" ratio for the different systems studied.

As explained in the Introduction, the purpose of this research is to be able to control the actuation extent, rate and time by means of different GT materials. As can be deduced from Figure 4, a wide range of materials, with different thermomechanical and structural properties, are used in the studied system; however, some requirements for the assembly process and the actuation must be considered. As explained in our previous work [25], in order to facilitate the assembling process and enhance the inter-layer adhesion in the actuator, the GT material should 
have a "thiol-epoxy" ratio close to or higher than the critical ratio in order to form a gelled structure after the $1^{\text {st }}$ curing stage (Figure $4(a)$ indicates the critical ratio $\left(r_{c}\right)$ of each system). In addition, the $T_{\mathrm{g}}$ of the final material (after the $2^{\text {nd }}$ curing stage) should be lower than $T_{\text {iso }}$ (in this case $T_{\text {iso }}$ is equal to $120^{\circ} \mathrm{C}$ ) to obtain an efficient actuation. Consequently, GT materials with $\mathrm{T}_{\mathrm{g}} \mathrm{S}$ ranging from well-below $T_{\text {iso }}$ to very close to $T_{\text {iso, }}$, were employed to fulfil these requirements, and to study the effect of both the GT network relaxation and LCN liquid-crystalline transition on the actuation, as well as the impact of the modulus and relaxation processes.

Keeping in mind all of the above, four different formulations are proposed. Table 2 summarizes the thermomechanical properties of these formulations. Two of the formulations have similar moduli (16.8 and $18.4 \mathrm{MPa}$ ) but different $\mathrm{T}_{\mathrm{gs}}\left(79\right.$ and $94{ }^{\circ} \mathrm{C}$ ), while the other two have similar $\mathrm{T}_{\mathrm{gS}}\left(118\right.$ and $120^{\circ} \mathrm{C}$ ), very close to $\mathrm{T}_{\text {iso }}\left(120^{\circ} \mathrm{C}\right)$, but with different moduli ( 37.4 and $42 \mathrm{MPa}$ ) and network relaxation dynamics (FWHM varies from 15.8 to $30.1{ }^{\circ} \mathrm{C}$ ).

Table 2. Thermomechanical properties of the different GT materials studied.

\begin{tabular}{cccccc}
\hline GT material & $\begin{array}{c}\mathbf{E}_{\mathbf{g}} \\
(\mathrm{MPa})\end{array}$ & $\begin{array}{c}\mathbf{E}_{\mathbf{r}} \\
(\mathrm{MPa})\end{array}$ & $\begin{array}{c}\mathbf{T}_{\mathbf{g}} \\
\left({ }^{\circ} \mathrm{C}\right)\end{array}$ & $\begin{array}{c}\text { tan } \boldsymbol{(} \\
\left({ }^{\circ} \mathrm{C}\right)\end{array}$ & $\begin{array}{c}\mathbf{F W H M} \\
\left({ }^{\circ} \mathrm{C}\right)\end{array}$ \\
\hline DGEBA-S3-60 & 2180 & 16.8 & 79 & 1.1 & 12.8 \\
\hline DGEBA-S4-60 & 2105 & 18.4 & 94 & 1.2 & 11.5 \\
\hline DGEBA-S4-35 & 2150 & 37.4 & 118 & 0.7 & 15.8 \\
\hline (DGEBA:SU8)-S3-35 & 2100 & 42.0 & 120 & 0.5 & 30.1 \\
\hline
\end{tabular}

Table 3 shows the specifications of the assembled actuators (dimension, LCN programmed strain, $\varepsilon_{\mathrm{p}}$, and volume fraction, $\mathrm{v}_{\mathrm{F}}$ ). For the sake of simplicity the nomenclature presented in Table 3 is slightly different from that used in our previous work [25]. In the present case, S3, S4 and SU8 refer to the GT material, and 60 or 35 refer to the "thiol-epoxy" ratio ( $r=0.6$ or $r=$ $0.35)$. In all the actuators, the volume fraction was fixed to 0.8 and the programming stress level to $2 \%$ of the corresponding stress at break $\left(\sigma_{b}\right)$. These parameters were fixed considering actuation extents lower than $60 \%$ (predicted by the model developed and presented in the previous work [25]), thus achieving quantifiable bending angles and deformations within the model limits. Moreover, the combination of $\mathrm{V}_{\mathrm{F}}=0.8$ and $\sigma_{\mathrm{p}}=2 \%$ leads to stiff and tough actuators, as observed in our previous work (neither failure nor layer delamination were observed for this configuration).

Table 3. Specifications of the different actuators studied. 


\subsection{Analysis of the cyclic actuation}

Before analysing the actuator response, all the actuators were subjected to 20 consecutive cycles to achieve a stable response. Figure 5 shows the cyclic analysis for the actuator AS360 as an example, since the evolution of the different parameters is comparable in terms of stability for all the actuators studied. The evolution of the actuation extent in $\%$ is presented as a function of the temperature and time in Figure $5(a)$ and (b), respectively. Bending and unbending temperatures $\left(T_{B}, T_{U}\right)$ and rates $\left(B_{\text {rate, }} U_{\text {rate }}\right)$ are shown in Figure $5(c)$ and $(d)$, respectively. The average value and the standard deviation of all the analysed parameters over 5 successive cycles were calculated to determine whether a sample had reached a steady behaviour. The parameter was considered to be stable when the standard deviation value was under $2 \%$, and this condition was repeated for at least five more consecutive cycles.

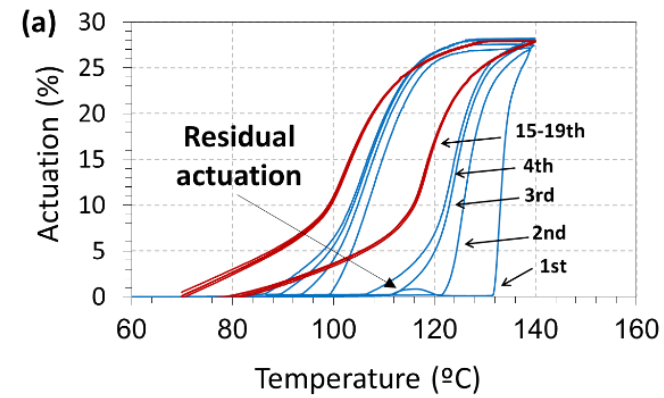

(c)

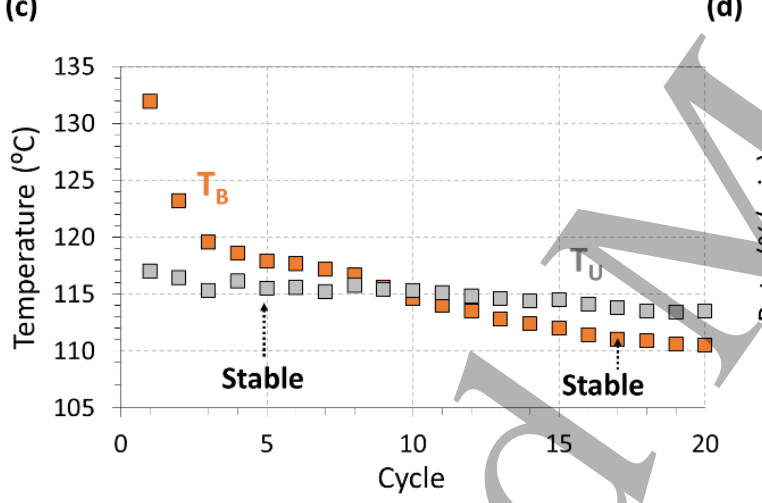

(d)
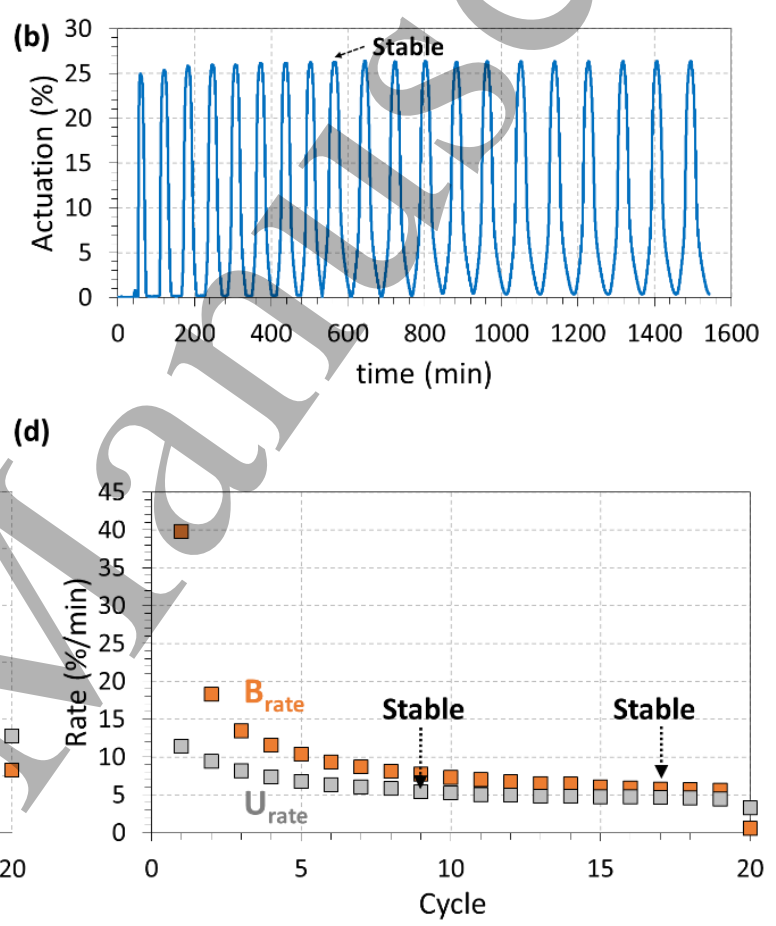

Figure 5. Stability analysis of actuator AS360: (a) Actuation-Temperature curves; (b) Actuation-time curves; (c) bending and unbending temperatures and (d) bending and unbending rates.

As can be observed in Figure $5(\mathrm{~b})$, the actuation extent increases slightly up to a stable value found after 9 cycles, and remains constant during the whole process. A similar response was found in our previous work [29] using an LCN (Di-C7) programmed at a considerably higher stress level. In [37] the mechanical response of the LCN showed an increase of the actuation up to a stable value achieved after 8 cycles and slightly decreased in the following cycles. Although the experimental conditions are not strictly comparable, in the present case, the initial period is very similar and the stability is extended further. This can be attributed to the state of the network structure after programming: the lower programming stress level in the LCN used for the actuator reduces the presence of internal stresses and unstable structural configurations.

Analysing the bending and unbending processes (Figure 5(c) and (d)) shows that all the parameters tend to decrease down to a stable value, but stabilization of the parameters corresponding to the bending process is more difficult. Both $B_{\text {rate }}$ and $T_{B}$ considerably decrease from the initial to the second cycle, whereas $U_{\text {rate }}$ and $T_{U}$ only show a slight decrease. Moreover, 
$B_{\text {rate }}$ and $T_{B}$ require more than 15 cycles to stabilize, whereas $U_{\text {rate }}$ and $T_{U}$ are stabilized after 5-9 cycles. The bending process, which is caused by the shrinkage of the LCN, is more affected during the first cycles and stabilizes later due to the structural rearrangements within the network of the LCN after the programming process, and probably due to the stress generated during the assembling process ( 2 nd curing stage of the GT). In contrast, unbending takes place after a heating procedure, and thus the effect regarding network instabilities is less relevant.

Overall, the width at half-height of the cycle narrows, the bending process starts earlier as deduced by the decreases in $\mathrm{T}_{\mathrm{B}}$ (see Figure $5(\mathrm{a})$ ), and the unbending process starts at a similar temperature; thus, the temperature range of the cycle is extended. Similarly, the decrease in the bending and unbending rates also extends the process in time. Moreover, it is worth noting that the first cycle shows a residual actuation before the bending process (highlighted in Figure $5(b)$ at $115^{\circ} \mathrm{C}$ ) and both the bending and unbending processes are steeper, which indicates a stress relaxation phenomenon. Various factors contribute to this response: On one hand, during the assembling process, residual stress is generated by the premature shrinkage of the LCN layer during epoxy homopolymerization. This causes an abrupt stress generation during the first heating procedure, and hence, the premature actuation. On the other hand, the LCN programming involves heating, deformation and cooling processes, and therefore, non-relaxed stress is probably present in the final network. This stress is accommodated after each heating procedure; thus, attenuating the drastic response during the bending process and in general, stabilizing the parameters related to the bending process.

The above results highlight that it is necessary to perform controlled cycling procedures to stabilize the actuators' responses before utilizing them to ensure controlled and repeatable operation.

\subsection{Effect of the GT structure}

Table 4 summarizes the first stable cycle (the cycle in which stability is accomplished for all the parameters studied), the stable values for the different parameters (Actuation extent, $T_{B}$, $T_{U}, B_{\text {rate }}, U_{\text {rate, }} t_{B}$ and $t_{U}$ ) and the analytical model prediction of the actuation extent (derived from our model developed in [25]).

Table 4. Stable actuation parameters for the different formulations studied

\begin{tabular}{|c|c|c|c|c|c|c|c|c|c|}
\hline Actuator & $\begin{array}{c}\text { Stable } \\
\text { Cycle }\end{array}$ & $\begin{array}{l}\text { Actuation } \\
\text { (\%) }\end{array}$ & $\begin{array}{c}\text { Actuation } \\
\text { Model } \\
(\%)\end{array}$ & $\begin{array}{c}T_{B} \\
\left({ }^{\circ} \mathrm{C}\right)\end{array}$ & $\begin{array}{l}T_{u} \\
\left({ }^{\circ} \mathrm{C}\right)\end{array}$ & $\begin{array}{c}B_{\text {rate }} \\
(\% / \text { min })\end{array}$ & $\begin{array}{c}\mathbf{t}_{\mathrm{B}} \\
(\mathrm{min})\end{array}$ & $\begin{array}{c}U_{\text {rate }} \\
(\% / \text { min })\end{array}$ & $\begin{array}{c}\mathbf{t u}_{u} \\
(\mathrm{~min})\end{array}$ \\
\hline $\operatorname{AS360}\left(T_{g}=79{ }^{\circ} \mathrm{C}\right)$ & 17 & 25.6 & 23.0 & 111.8 & 114.2 & 6.3 & 11.8 & 5.1 & 14.9 \\
\hline AS460 $\left(T_{g}=94^{\circ} \mathrm{C}\right)$ & 19 & 23.9 & 21.0 & 115.0 & 120.1 & 6.3 & 11.1 & 5.4 & 13.5 \\
\hline AS435 $\left(T_{g}=118^{\circ} \mathrm{C}\right)$ & 18 & 12.3 & 11.0 & 120.2 & 119.5 & 9.8 & 7.3 & 7.3 & 9.7 \\
\hline ASU835 $\left(T_{g}=120^{\circ} \mathrm{C}\right)$ & 19 & 5.7 & 8.2 & 121.8 & 122.9 & 12.3 & 6.9 & 8.7 & 9.9 \\
\hline
\end{tabular}

First, it is worth noting that the actuation extent of these actuators fits very well with the predictions of the analytical model developed in our previous work [25]. The deviation is about $10 \%$ in all cases except for sample ASU835, in which the deviation is higher, probably due to a post-curing process after the first actuation cycle that altered the initial model predictions. In general, the model can predict the maximum actuation extent considering the actuator 
configuration (geometrical relation between the layers), material modulus and the programmed strain extent of the LCN. In the present case, the modulus of the GT and its relaxation dynamics varied, whereas the geometrical design $\left(\mathrm{V}_{\mathrm{F}}\right)$ and the LCN programming stress level were kept constant. It was found that the actuation extent decreased when the rubbery modulus was increased: from 16.8 to $18.4 \mathrm{MPa}$ (AS360 and AS460) the actuation shows comparable values, $25.6 \%$ and $23.9 \%$, whereas at $37 \mathrm{MPa}$ (AS435) it drops down to $12.3 \%$ and at $42 \mathrm{MPa}$ (ASU835) to $5.7 \%$. This suggests that the key factor for determining the maximum actuation extent is the GT material modulus rather than the $T_{\mathrm{g}}$ or the structural relaxation of the GT and LCN materials. This makes it possible to exploit the effect of varying the relaxation dynamics of the GT network without affecting the actuation extent.

Analysing the bending and unbending temperatures it can be seen that both stabilize at a temperature very close to $\mathrm{T}_{\text {iso }}\left(\mathrm{T}_{\text {iso }}=120^{\circ} \mathrm{C}\right)$, indicating that the actuation takes place within the LCN liquid-crystalline transition. However, both $T_{B}$ and $T_{U}$ increase with increasing $T_{g} s$ of the GT. As $T_{\mathrm{g}}$ approaches $T_{\text {iso, }}$, the relaxation of the GT network takes place later. When $T_{\mathrm{g}}$ is very close to $T_{\text {iso }}$ (formulations AS435 and ASU835), $T_{B}$ is inherently displaced to higher values due to the GT material impeding LCN shrinkage until relaxation takes place. Similarly, the unbending process takes place earlier due to the coupling of the GT vitrification with the LC transition on cooling down. Although AS435 has the same $T_{\mathrm{g}}$ as ASU835, this effect is accentuated in the formulation with SU8 probably because of the broader relaxation process that delays the processes towards higher temperatures. Therefore, the beginning of both the bending and unbending processes depends not only on the LC transition of the LCN, but also on the GT relaxation when coupling between structural transitions takes place.

However, bending and unbending rates tend to increase on increasing the $T_{g}$, suggesting that the processes are faster for the formulations with higher $T_{\mathrm{g}}$. This is in accordance with the decrease in $t_{B}$ and $t_{u}$. Formulations with higher $\mathrm{T}_{\mathrm{g}}$ have broader relaxation processes (mostly the formulation containing SU8); thus, slower bending-unbending processes would be expected. Nevertheless, if the GT relaxation takes place at a temperature close to or above $T_{\text {iso, }}$, the LCN shrinkage is initially impeded by the high modulus of the GT; thus, when it finally takes place, the force driven by the LCN is higher and the process becomes more drastic and faster. 
(a)

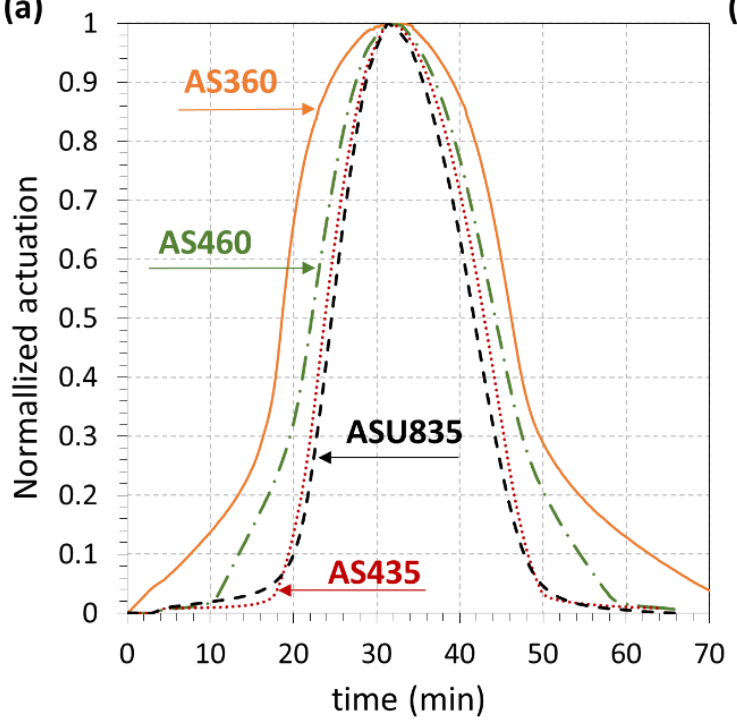

(b)

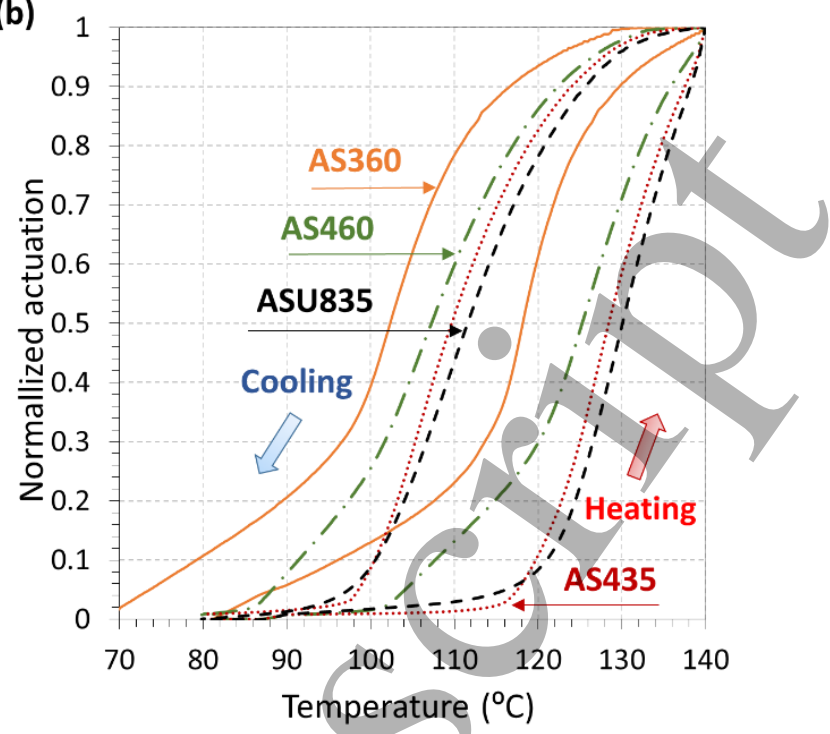

Figure 6. Normalized actuation as a function of time (a) and temperature (b) for the different formulations studied at a heating and cooling rate of $2^{\circ} \mathrm{C} / \mathrm{min}$.

Figure 6 shows the stable cycles for all the actuators studied. To be able to make comparisons among the different formulations and actuation extent levels, the curves have been normalized to the actuation extent and presented as a function of time (Figure 6(a)) and temperature (Figure $6(b))$. As stated above, the increase in $T_{g}$ leads to faster bending and unbending processes. This can be seen clearly in the actuation-time cycles in Figure 6(a). The formulations with a $T_{g}$ close to $T_{\text {iso }}$ have a narrower cycle, as the total time is considerably reduced due to the shift of $T_{B}$ and $T_{u}$ to higher and lower temperatures, respectively. This implies later bending and earlier unbending processes, coupled with the drastic response of the LCN when the shrinkageelongation processes are triggered, which can be deduced by the increase in the slope in both steps. Moreover, in Figure $6(\mathrm{~b})$, the effect of the $L C N$ response on increasing the $T_{g}$ can be clearly seen: there is a two-step actuation process with a lower slope initial part that takes place at 80 ${ }^{\circ} \mathrm{C}$ for AS360, which is shifted to $100^{\circ} \mathrm{C}$ for AS460 and almost disappears for the AS435 and ASU835 actuators. This early actuation is connected to the glass transition of the GT material that is $79^{\circ} \mathrm{C}$ in AS360 and $94^{\circ} \mathrm{C}$ in AS460. The softening of the network allows a progressive bending associated with the relaxation of the LCN network on approaching $\mathrm{T}_{\text {iso. When }}$ isotropization takes place the slope increases, which indicates LCN shrinkage. In contrast, when the $T_{g}$ of the GT material is close to $T_{\text {iso, }}$ this relaxation taking place in the LCN cannot be accommodated in the shape of bending due to the high modulus of the GT material and it is accumulated in terms of energy that is eventually released when the GT relaxes, hence the sharper response.

\subsection{Effect of the heating rate}

As evidenced in the previous section, the GT relaxation has a crucial impact on the actuation response. It is well known that the network relaxation dynamics are affected by the heating rate [32]; therefore, the response of the actuators to different heating rates was studied. First, the study of actuator AS360 is presented in Figure 7(a) with the Actuation-time cycles (blue-line) at different heating rates (from $0.2{ }^{\circ} \mathrm{C} / \mathrm{min}$ to $20 \stackrel{\circ}{\circ} / \mathrm{min}$, with constant cooling rate of $2^{\circ} \mathrm{C} / \mathrm{min}$ ) 
performed in a single cycling experiment. The variation in temperature during the whole experiment is represented in this figure with a red dotted line. In Figure 7(b) and (c) the bending parameters, $B_{\text {rate }}$ and $t_{B}$, are also shown for all the formulations studied. It must be noted that from a rate of $5{ }^{\circ} \mathrm{C} / \mathrm{min}$ to $20^{\circ} \mathrm{C} / \mathrm{min}$ the holding time at a high temperature (during bending) was extended from 3 to $30 \mathrm{~min}$ to allow the completion of the process due to the rapid increase in the temperature in the thermal chamber of the DMA and the thermal inertia of the sample.
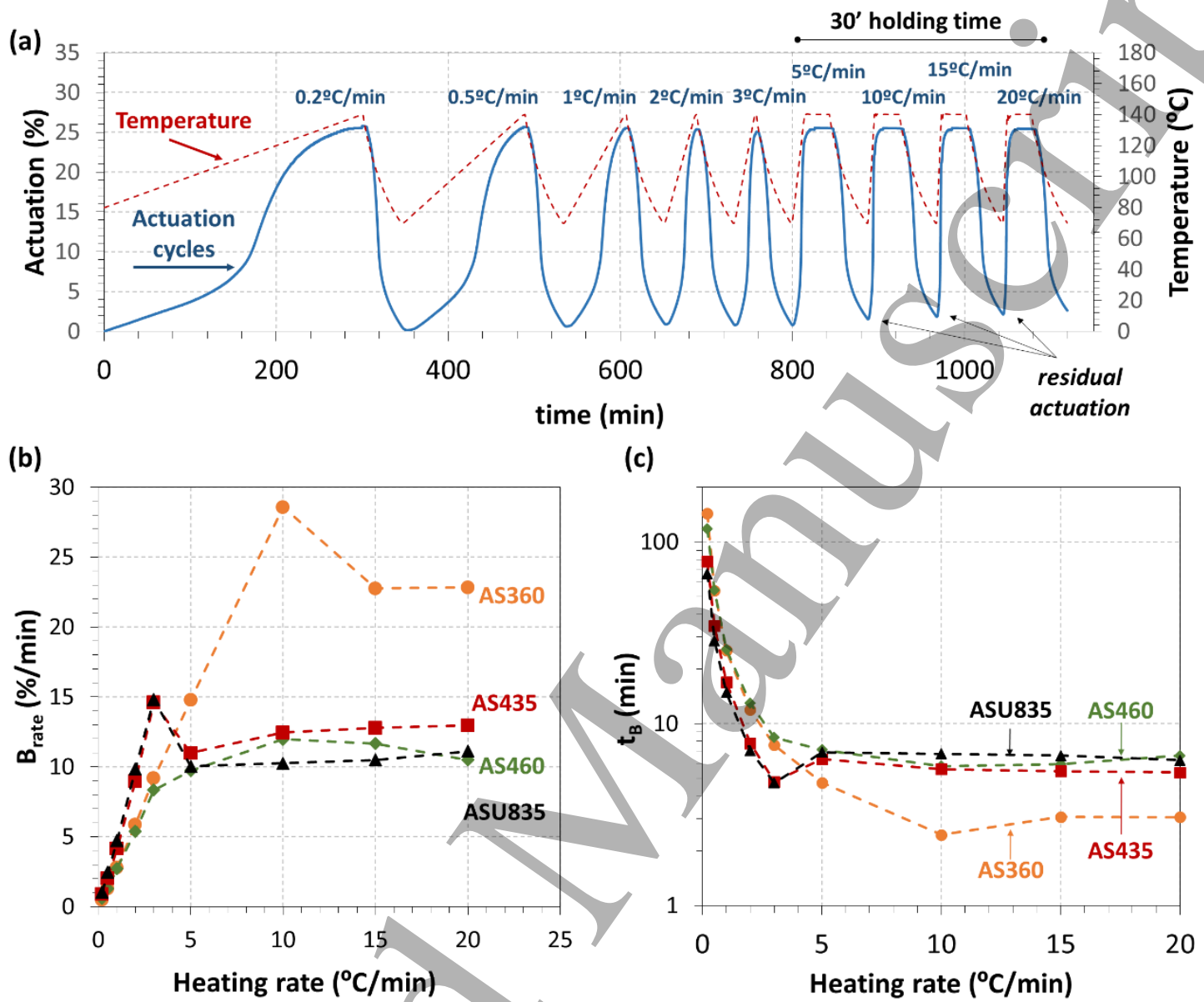

Figure 7. Effect of the heating rate on the actuation behaviour: (a) Actuation-time-Temperature cycles in actuator AS360, (b) bending rate and (c) bending time at different heating rates for the different formulations studied.

Overall, on increasing the heating rate from $0.2^{\circ} \mathrm{C} / \mathrm{min}$ to $3^{\circ} \mathrm{C} / \mathrm{min}$, the cycles tend to narrow. The early bending due to $L C N$ relaxation above $T_{g}$ and below $T_{\text {iso, }}$, and the further bending caused by the LCN shrinkage overlap, and hence the actuation becomes sharper. Moreover, the actuation extent is not affected. If we analyse the cycles with an extended time at a high temperature (heating rates from 5 to $20^{\circ} \mathrm{C} / \mathrm{min}$ ) for the AS360 formulation (Figure 7(a)), it can be seen that the actuation extent is not affected. However, the capability to recover the original flat shape is slightly worsened as deduced by the appearance of the residual actuation (indicated with an arrow in Figure 7(a)). This could be related to the creep phenomenon observed after each shrinkage-elongation cycle of the LCN in our previous work [29]. When the LCN was under free actuation conditions, the creep was attributed to irrecoverable or plastic deformations taking place during each cycle. In the present case, the LCN motion is completely impeded; therefore, the LCN cannot suffer creep, but rather stress relaxation. During the 20 consecutive cycles, the actuator performance was excellent; however, if the time at a high temperature is 
increased, the LCN network can suffer stress-relaxation, leading to a new "stable" state that loses the original "flat" shape taking on a new slightly bent shape promoted by the bending actuation design.

Analyses of the bending parameters (see Figure $7(b)$ and $7(c)$ ) for the AS360 formulation show that there is a clear acceleration of the bending process with the increase of the heating rate associated with the enhancement of the relaxation dynamics in both the GT and LCN networks. However, a maximum appears at $10^{\circ} \mathrm{C} / \mathrm{min}$ and this acceleration is attenuated and stabilized at $15^{\circ} \mathrm{C} / \mathrm{min}$. This stable value is related to the coupling of the LCN shrinkage and network relaxation of the GT. When the heating rate is too high, the temperature in the sample is not homogeneous: the outer faces have a higher temperature than the inner ones. Therefore, the LCN layer, which is placed in the outer face, begins the shrinkage process before or while the GT relaxation is taking place. As a result, the shrinkage is impeded and the process is controlled by the relaxation of the GT network. This phenomenon is closely related to the explanation given above regarding the proximity of the $T_{\mathrm{g}}$ with $\mathrm{T}_{\text {iso. }}$. In this case, both processes take place simultaneously, hence a coupling effect is noticed. In the present case, the delay in the GT relaxation when the heating rate is too high causes the same effect. This suggests that the actuation can be controlled with the heating rate by the relaxation of the GT network or by the LCN shrinkage-elongation processes. Moreover, the coupling of the GT relaxation with the LCN shrinkage can promote stress-relaxation due to the constraining forces that are generated, favouring the residual actuation observed in those cycles with an extended time at a high temperature (Figure 7(a)). To avoid this stress-relaxation phenomenon, it is of outmost importance to control the time at a high temperature and it must fit within the necessary time to fully actuate during the bending.

Comparing $B_{\text {rate }}($ Figure $7(b))$ and $t_{B}$ (Figure $\left.7(c)\right)$ of the different formulations studied shows that the explanation given above regarding the faster bending and unbending processes in formulations with higher $T_{g}$ is corroborated when low heating rates are imposed (from 0.2 to 3 $\left.{ }^{\circ} \mathrm{C} / \mathrm{min}\right)$. In this case, $\mathrm{T}_{\mathrm{g}}$ closer to $\mathrm{T}_{\text {iso, }}$ faster $\mathrm{B}_{\text {rate }}$ and lower $\mathrm{t}_{\mathrm{B}}$. However, on increasing the heating rate, both $B_{\text {rate }}$ and $t_{B}$ are attenuated and stabilized earlier for those formulations with a $T_{g}$ close to $\mathrm{T}_{\text {iso }}$ (AS435 and ASU835 stabilizes at $5^{\circ} \mathrm{C} / \mathrm{min}$ ). This can be rationalized as follows: when the heating rate increases, the network relaxation of the GT governs the bending process due to the coupling of both the $\mathrm{LC}$ and glass transitions. This phenomenon occurs earlier in formulations with a $T_{\mathrm{g}}$ close to $\mathrm{T}_{\text {iso }}$ (the coupling effect was observed at $2{ }^{\circ} \mathrm{C} / \mathrm{min}$ in Table 4 and Figure 6 ). Under coupling conditions, the drastic response caused by the impeded shrinkage of the LCN seems to be affected by the nature of the network structure: formulations with S4 (AS460 and AAS435) show similar behaviour with respect to $B_{\text {rate }}$ and $t_{B}$ probably because of the more homogeneous network structure in comparison with AS360 with the presence of a side ethyl chain. However, the presence of SU8 in the S3 system attenuates the effect of the ethyl chain due to the broadening of the relaxation process.

In conclusion, the modulus of the materials and the configuration of the actuator dictate the actuation extent, whereas the dynamics of the network relaxation in both the GT and LCN materials can be used to tailor both the rate and total time needed for the actuation process. The actuation rate can be tailored as desired by implementing the appropriate heating procedure, rapid heating to promote the coupling of the two transitions, and hence control the GT relaxation, that is, isothermal processes at a high temperature, or slower heating to control through the LCN shrinkage-elongation process. Moreover, controlling by GT relaxation means 
that no early bending takes place and that the actuator stiffness is preserved until the bending process is triggered; thus, the mechanical capabilities during actuation are enhanced. However, controlling by the LCN leads to early bending and softening of the material so that it can be used in soft robotics, for instance as a soft clamp mechanism to avoid damaging delicate objects.

\subsection{Development of a 3D actuating device}

To demonstrate the capability of these materials controlled in terms of actuation rate and time, a device made of differently designed actuators was developed in one single element following the curing procedure explained in the Experimental Section. The top and side views of the device with the specific dimensions are shown in Figure 8. The code of the different GT materials that form the device have been highlighted in the top view (Figure 8(a)), as well as the code corresponding to each leg. In addition, the configuration of leg (6) has been highlighted (Figure $8(\mathrm{c})$ ) to better understand the position of each LCN and GT material to achieve the "Stype" motion. All the LCNs were programmed at the same level $\left(\sigma_{P}\right)$.

(a)

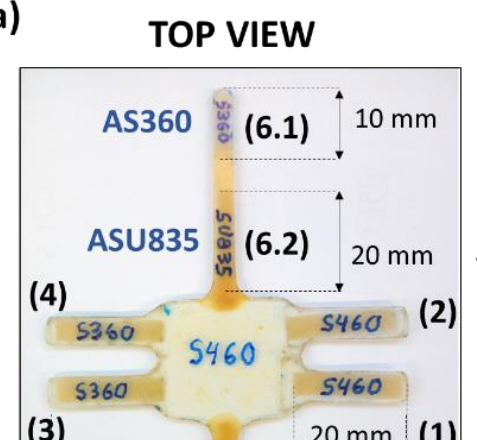

(b)

Figure 8. (a) Top view of the device after the assembling process: the GT materials (notation in blue), the nomenclature (numbers in black and in brackets) and dimensions of the legs have been included; (b) side view of the device (the side is indicated by an arrow in (a)); (c) Zoom of the side view of the central portion of leg (6) highlighting the materials and the configuration used in this leg.

It can be observed in the TOP view that the device has different colorations depending on the GT material. Darker coloration in the AS435 and ASU835 systems (see legs 5-6) is an indication of a higher contribution of epoxy homopolymerization initiated by $1 \mathrm{MI}$, as observed in our previous work [27]. The proposed methodology for the adhesion and assemblage of each leg led to a compact device without superficial defects or scratches and a constant thickness of $1.2 \mathrm{~mm}$, as observed in the side view. The different GT materials were properly merged (ensuring a solid connection) during the 2 nd curing stage as evidenced by the diffusion of the coloured and transparent materials in the joints between the central part and the legs. 


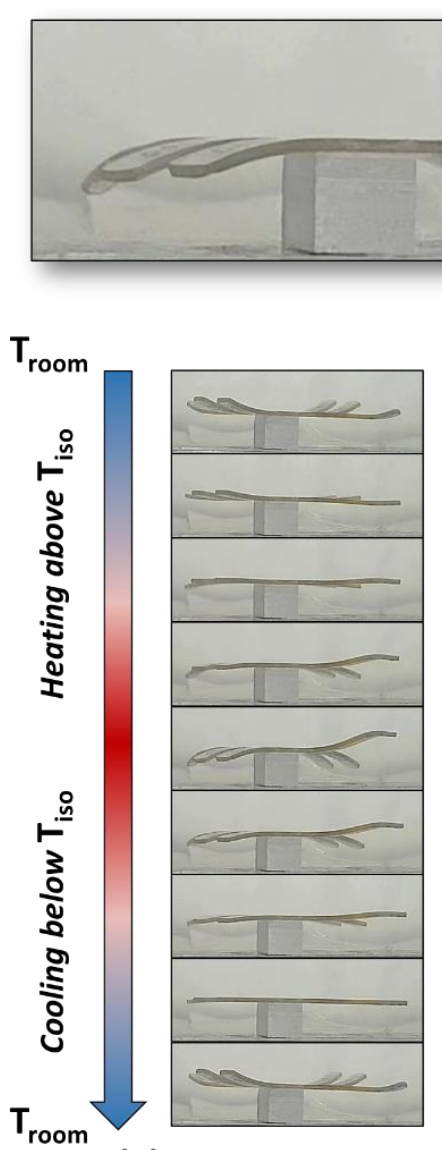

(a) Perspective view
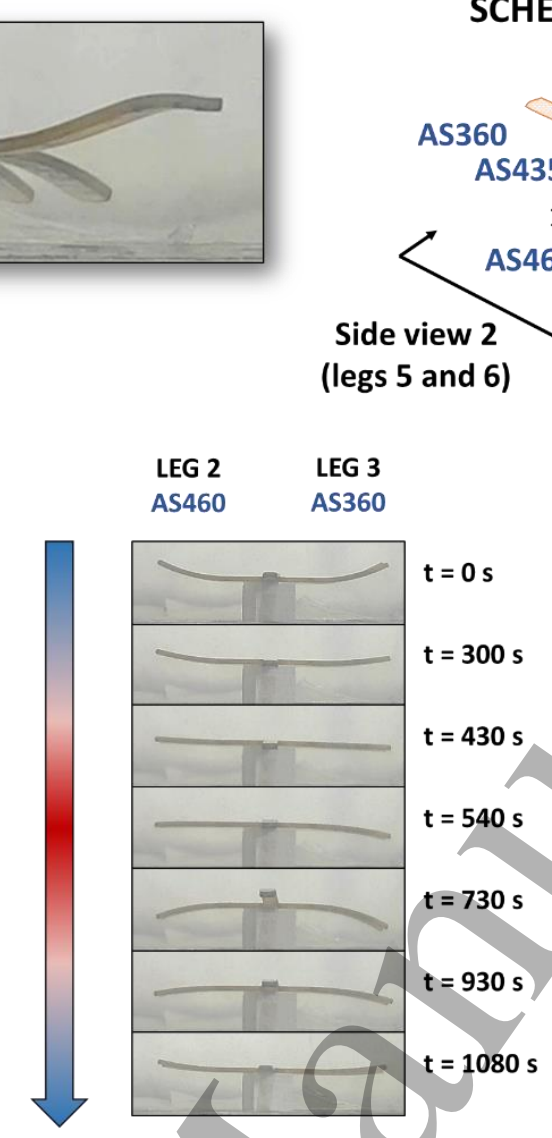

(b) Side view 1

\section{SCHEME OF THE VIEWS}

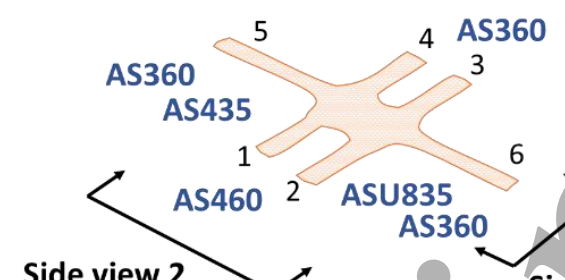

Side view 1

(legs 2 and 3)
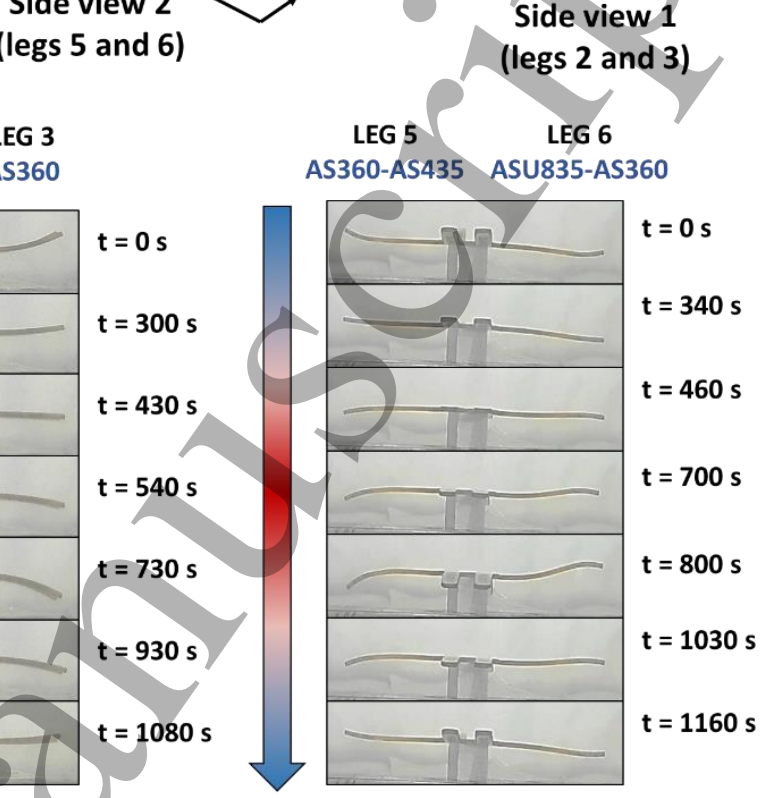

(c) Side view 2

Figure 9. Visual demonstration of the complex device motion from different perspectives and side views under heating-cooling procedures.

The actuation under heating-cooling procedures is shown in Figure 9, which displays the different actuation levels achieved from each actuator (the actuators are named "legs" in Figure 9 according to the nomenclature used in Figure 8). The scheme of the device and the testing views are shown on top. In the first set of frames (Figure 9(a)), a perspective view of the actuation response is shown for a rapid heating process above $\mathrm{T}_{\text {iso }}$ (first photograph-middle photograph) and subsequent natural cooling below $\mathrm{T}_{\text {iso }}$ (middle photograph-last photograph) after 20 previous cycles (stabilization of all the actuators). First, it is worth noting that the device starts from an already bent shape. As explained in the previous work [25], after the assembling process, the internal stress generated during the curing process and premature shrinkage of the LCN leads to some curvature in the opposite direction to the actuation. Moreover, this curing process was carried out at a high temperature (above $T_{\text {iso }}$ ), and thus this effect was accentuated. This can be addressed by using compounds which absorb and dissipate energy during the reaction, such as hyperbranched polymers [32]. However, it is possible to take advantage of this for processing curved shapes from simple planar designs.

Analysing the motion of the device in detail (Figure 9(b) and (c)), it can be seen that the designed motions have been successfully achieved: legs (2) and (3) bend downwards (and legs (1) and (4) that are behind in Figure 9(b)), whereas leg (6) bends shaping an "S" and leg (5) remains straight and bends downwards only in section (5.1). This is repeated with no appreciable loss of shape, as observed in the stability section. As explained in the previous section, when 
rapid heating is applied, the GT relaxation controls the process in all the actuators; thus, the narrower the GT relaxation, the faster the actuation. In addition, the presence of the S3 should accentuate this acceleration. Therefore, the expected motion sequence should follow the order: AS360 > AS460 > ASU835. This can be observed in Figure 9(b), in the side view of legs 2-3, where leg (3) (and leg (4) behind) made of AS360 bend earlier than leg (2) (and leg (1) behind) made of AS460, as deduced in the progressive bending from frames 0 to frame $540 \mathrm{~s}$. This becomes clearer if legs (5) and (6) in Figure 9(c) are analysed, where ASU835 (section 6.2 in leg 6) can be compared to AS360 (section 6.1 and 5.1). As can be seen, after 460 seconds AS360 has almost completed the bending process, whereas ASU835 has just started to bend. The premature beginning of AS360 in section (6.1) is better appreciated in leg (5), section (5.1), because section (5.2) has no LCN (static section); thus, no coupling of the bending processes takes place. At 700 $s$ both ends of the legs (6.1 and 5.1) have almost finished bending, whereas 6.2 (made of ASU835) is starting to bend and finishes rapidly at $800 \mathrm{~s}$ due to the drastic response. After the bending process, during the cooling step the process reverts back: ASU835 unbending takes place first (see frame at $1030 \mathrm{~s}$ ) and AS360 starts later (see frame at $1160 \mathrm{~s}$ ) because of the higher $T_{\mathrm{g}}$ of ASU835: as observed in the previous sections, the bending process is delayed by approaching $\mathrm{T}_{\mathrm{g}}$ to $\mathrm{T}_{\text {iso, }}$, but the unbending takes place earlier.

Side view (legs 1-4)

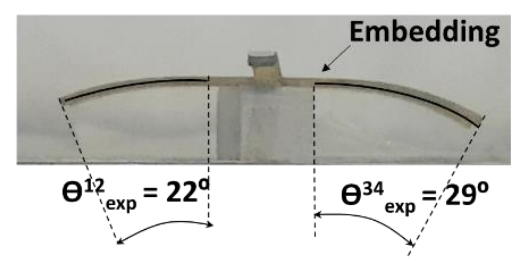

Side view (legs 5-6)

\section{Model predictions:}

Geometrical input:

$\mathrm{t}_{11}=0.24 \mathrm{~mm}$

- $\mathrm{t}_{\mathrm{L} 2}=0.235 \mathrm{~mm} \quad \mathrm{v}_{\mathrm{F}}=0.8$

- $\mathrm{t}_{\mathrm{L} 3}=0.73 \mathrm{~mm}$

Model output (bending angle):

$\theta_{\text {model }}^{12}=29^{\circ} / / \theta_{\text {exp }}^{12}=22^{\circ} \quad\left(E_{r}=16.8 \mathrm{MPa}, L=20 \mathrm{~mm}\right)$

$\theta_{\text {model }}^{43}=32^{\circ} / / \theta^{43}{ }_{\text {exp }}=29^{\circ} \quad\left(E_{\mathrm{r}}=18.4 \mathrm{MPa}, \mathrm{L}=20 \mathrm{~mm}\right)$

$\theta^{5.1}$ model $=16^{\circ} / / \theta_{\text {exp }}^{51}=22^{\circ} \quad\left(E_{r}=16.8 \mathrm{MPa}, L=10 \mathrm{~mm}\right)$

$\theta^{6.1}{ }_{\text {model }}=16^{\circ} / / \theta_{\text {exp }}^{61}=19^{\circ}\left(E_{\mathrm{r}}=16.8 \mathrm{MPa}, \mathrm{L}=10 \mathrm{~mm}\right)$

$\theta^{6.2}{ }_{\text {model }}=15^{\circ} / / \theta_{\text {exp }}^{62}=19^{\circ}\left(E_{r}=42 \mathrm{MPa}, L=20 \mathrm{~mm}\right)$

Figure 10. Determination of the bending angles (actuation) in each leg and comparison with the model predictions.

In Figure 10, the actuation extent of each actuator in the device has been experimentally calculated and compared with the predictions of the analytical model developed in our previous work [25]. As it can be seen, the experimental actuation extent is slightly lower in legs (1) and (4) in comparison to the model predictions, whereas in legs (5) and (6) the tendency is the opposite, the experimental values are slightly higher. In general, the experimental and predicted values fit very well considering the experimental limitations for assembling such a complex device (the positioning of the LCN layers and the adjustment of the width, length and thickness of each part of the device). Moreover, in the model the actuator was analysed in free bending (with only two supports), whereas in the present case it can be considered as a cantilever beam with an embedded part in the joint between the legs and the central part (highlighted in Figure 10). These different boundary conditions produce slightly lower bending angles (legs 1-4), and coupled bending in leg (6), and thus higher actuation, which makes it difficult to experimentally determine the bending angle. 


\section{CONCLUSIONS}

In this work, glassy thermosets with different thermomechanical and structural properties and a liquid-crystalline network with high $\mathrm{T}_{\text {iso }}$ have been used to develop free-standing shapememory actuators with enhanced control of the actuation in terms of extent, rate and time. The relevance of the dual-curing processing for the assembling and precise control of the final structural properties and configuration of the actuators has been demonstrated. The actuators have shown good stability on cycling with a stabilization extent of 17-19 cycles to accommodate internal stresses generated during the assembling process and programming of the LCN.

The use of GT materials with different moduli makes it possible to control the actuation extent according to the predictions of our analytical model. Moreover, when GT materials with a $T_{g}$ closer to the $T_{\text {iso }}$ of the LCN are used, a coupling effect between the network relaxation of GT and LC transition is evidenced. As a consequence, it is possible to control the actuation with the GT network relaxation. Furthermore, the heating rate can induce this coupling effect: at low heating rates, only formulations with $\mathrm{T}_{\mathrm{g}} \approx \mathrm{T}_{\text {iso }}$ show the coupling effect; therefore, the actuation is controlled by the LCN shrinkage-elongation process. However, if the heating rate is high enough, the coupling effect takes place even with $T_{\mathrm{g}}<T_{\text {iso }}$ because of the presence of temperature gradients within the sample and delayed network relaxation. This leads to the actuation being controlled by the GT network relaxation.

In addition, in this work the possibility of tuning the actuation extent, rate and time for the proposed actuators has been experimentally demonstrated by designing and manufacturing a 3D actuating device consisting of different actuator configurations and able to make different motions at different times. The proposed assembling methodology has made it possible to develop a compact 3D design in one single piece with a controllable soft motion that fitted the model predictions very well.

\section{ASSOCIATED CONTENT}

Supporting Information. The molecular structures of all the compounds studied in the manuscript are available in the supporting information. An illustrative video of the actuation response for the 3D device designed is also available.

\section{ACKNOWLEDGMENT}

The authors would like to thank MINECO (MAT2017-82849-C2-1-R and MAT2017-82849-C2-2R) and Generalitat de Catalunya (2017-SGR-77) for financial support. Xavier F.F. also acknowledges the Serra Húnter programme from the Generalitat de Catalunya.

\section{REFERENCES}

[1] M. Behl, A. Lendlein, Actively moving polymers, Soft Matter. 3 (2007) 58-67. doi:10.1039/B610611K.

[2] A. Lendlein, C. Wischke, K. Kratz, M. Heuchel, J. Zotzmann, B. Hiebl, A.T. Neffe, M. Behl, B. Development, Shape-Memory Polymers, Elsevier Ltd., 2011. doi:10.1016/B978-0-08055294-1.00271-3. 
[3] X. Wu, W.M. Huang, Y. Zhao, Z. Ding, C. Tang, J. Zhang, Mechanisms of the shape memory effect in polymeric materials, Polymers. 5 (2013) 1169-1202. doi:10.3390/polym5041169.

[4] Q. Zhao, H.J. Qi, T. Xie, Recent progress in shape memory polymer: New behavior, enabling materials, and mechanistic understanding, Prog. Polym. Sci. 49-50 (2015) 1-42. doi:10.1016/j.progpolymsci.2015.04.001.

[5] C. Ohm, M. Brehmer, R. Zentel, Liquid crystalline elastomers as actuators and sensors, Adv. Mater. 22 (2010) 3366-3387. doi:10.1002/adma.200904059.

[6] J. Sun, Q. Guan, Y. Liu, J. Leng, Morphing aircraft based on smart materials and structures: A state-of-the-art review, J. Intell. Mater. Syst. Struct. 27 (2016) 2289-2312. doi:10.1177/1045389X16629569.

[7] C.L. Lewis, Y. Meng, M. Anthamatten, Well-Defined Shape-Memory Networks with High Elastic Energy Capacity, Macromolecules (2015) 150715075734002. doi:10.1021/acs.macromol.5b00763.

[8] X. Xiao, D. Kong, X. Qiu, W. Zhang, F. Zhang, L. Liu, Y. Liu, S. Zhang, Y. Hu, J. Leng, ShapeMemory Polymers with Adjustable High Glass Transition Temperatures, Macromolecules. 48 (2015) 150521152304000. doi:10.1021/acs.macromol.5b00654.

[9] R. Biju, C.P.R. Nair, Synthesis and characterization of shape memory epoxy-anhydride system, J. Polym. Res. 20 (2013) 82. doi:10.1007/s10965-013-0082-z.

[10] N. Zheng, G. Fang, Z. Cao, Q. Zhao, T. Xie, High strain epoxy shape memory polymer, Polym. Chem. 6 (2015) 3046-3053. doi:10.1039/C5PY00172B.

[11] A. Belmonte, D. Guzmán, X. Fernández-Francos, S. De la Flor, Effect of the Network Structure and Programming Temperature on the Shape-Memory Response of ThiolEpoxy “Click" Systems, Polymers. 7 (2015) 2146-2164. doi:10.3390/polym7101505.

[12] A. Belmonte, X. Fernández-Francos, S. De la Flor, New understanding of the shapememory response in thiol-epoxy click systems : towards controlling the recovery process, J. Mater. Sci, 52 (2017) 1625-1638. doi:10.1007/s10853-016-0456-9.

[13] D. Santiago, A. Fabregat-Sanjuan, F. Ferrando, S. De la Flor, Recovery stress and work output in hyperbranched poly(ethyleneimine)-modified shape-memory epoxy polymers, J. Polym. Sci. Part B Polym. Phys. 54 (2016) 1002-1013. doi:10.1002/polb.24004.

[14] S. Pandini, F. Bignotti, F. Baldi, S. Passera, Network architecture and shape memory behavior of cold-worked epoxies, J. Intell. Mater. Syst. Struct. 24 (2013) 1583-1597. doi:10.1177/1045389X13478275.

[15] A. Belmonte, X. Fernández-Francos, S. De la Flor, À. Serra, Network structure dependence on unconstrained isothermal-recovery processes for shape-memory thiol-epoxy "click" systems, Mech. Time-Dependent Mater. 21 (2017) 133-149. doi:10.1007/s11043-0169322-z.

[16] Y. Li, C. Pruitt, O. Rios, L. Wei, M. Rock, J.K. Keum, A.G. Mcdonald, M.R. Kessler, Controlled shape memory behavior of a smectic main-chain liquid crystalline elastomer, Macromolecules. 48 (2015) 2864-2874. doi:10.1021/acs.macromol.5b00519. 
[17] K.A. Burke, I.A. Rousseau, P.T. Mather, Reversible actuation in main-chain liquid crystalline elastomers with varying crosslink densities, Polymer. 55 (2014) 5897-5907. doi:10.1016/j.polymer.2014.06.088.

[18] G.C. Lama, P. Cerruti, M. Lavorgna, C. Carfagna, V. Ambrogi, G. Gentile, Controlled Actuation of a Carbon Nanotube/Epoxy Shape-Memory Liquid Crystalline Elastomer, J. Phys. Chem. C. 120 (2016) 24417-24426. doi:10.1021/acs.jpcc.6b06550.

[19] J. Zhou, S.S. Sheiko, Reversible shape-shifting in polymeric materials, J. Polym. Sci. Part B Polym. Phys. 1 (2016) 1-16. doi:10.1002/polb.24014.

[20] C.L. Lewis, E.M. Dell, A Review of Shape Memory Polymers Bearing Reversible Binding Groups, J. Polym. Sci. Part B Polym. Phys. 54 (2016) 1340-1364. doi:10.1002/polb.23994.

[21] Y. Meng, J. Jiang, M. Anthamatten, Shape actuation via internal stress-induced crystallization of dual-cure networks, ACS Macro Lett. 4 (2015) 115-118. doi:10.1021/mz500773v.

[22] M.O. Saed, A.H. Torbati, D.P. Nair, C.M. Yakacki, Synthesis of Programmable Main-chain Liquid-crystalline Elastomers Using a Two-stage Thiol-acrylate Reaction, J. Vis. Exp. 107 (2016) 1-10. doi:10.3791/53546.

[23] K.K. Westbrook, P.T. Mather, V. Parakh, M.L. Dunn, Q. Ge, B.M. Lee, H.J. Qi, Two-way reversible shape memory effects in a free-standing polymer composite, Smart Mater. Struct. 20 (2011) 65010. doi:10.1088/0964-1726/20/6/065010.

[24] M. Zarek, M. Layani, I. Cooperstein, E. Sachyani, D. Cohn, S. Magdassi, 3D Printing of Shape Memory Polymers for Flexible Electronic Devices, Adv. Mater. 28 (2016) 44494454. doi:10.1002/adma.201503132.

[25] A. Belmonte, G.C. Lama, G. Gentile, P. Cerruti, V. Ambrogi, X. Fernández-Francos, S. De la Flor, Thermally-triggered free-standing shape-memory actuators, Eur. Polym. J. (2017). doi:10.1016/j.eurpolymj.2017.10.006.

[26] X. Fernández-Francos, A.-O. Konuray, A. Belmonte, S. De la Flor, À. Serra, X. Ramis, Sequential curing of off-stoichiometric thiol-epoxy thermosets with a custom-tailored structure, Polym. Chem. 7 (2016) 2280-2290. doi:10.1039/C6PY00099A.

[27] A. Belmonte, X. Fernández-Francos, À. Serra, S. De la Flor, Phenomenological characterization of sequential dual-curing of off-stoichiometric " thiol-epoxy " systems: Towards applicability, Mater. Des. 113 (2017) 116-127. doi:10.1016/j.matdes.2016.10.009.

[28] A. Belmonte, C. Russo, V. Ambrogi, X. Fernández-Francos, S. De la Flor, Epoxy-based shape-memory actuators obtained via dual-curing of off-stoichiometric "thiol-epoxy" mixtures, Polymers. 9 (2017) 113. doi:10.3390/polym9030113.

[29] A. Belmonte, G.C. Lama., G. Gentile, X. Fernández-Francos, S. De la Flor, P. Cerruti, V. Ambrogi, Synthesis and Characterization of Liquid-Crystalline Networks: Towards Autonomous Shape-Memory Actuation, J. Phys. Chem. C. 121 (2017) 22403-22414. doi:10.1021/acs.jpcc.7b04610.

[30] C.-H. Hsueh, Modeling of elastic deformation of multilayers due to residual stresses and 
external bending, J. Appl. Phys. 91 (2002) 9652-9656.

[31] J. Malzbender, Mechanical and thermal stresses in multilayered materials, J. Appl. Phys. 95 (2004) 1780-1782. doi:10.1063/1.1642289.

[32] C. Acebo, M. Alorda, F. Ferrando, X. Fernández-Francos, A. Serra, J.M. Morancho, J.M. Salla, X. Ramis, Epoxy/anhydride thermosets modified with end-capped star polymers with poly(ethyleneimine) cores of different molecular weight and poly( $\varepsilon$-caprolactone) arms, Express Polym. Lett. 9 (2015) 809-823. doi:10.3144/expresspolymlett.2015.76. 